\title{
Detection of the hydrogen Balmer lines in the ultra-hot Jupiter WASP-33b
}

\author{
F. Yan ${ }^{1}$, A. Wyttenbach ${ }^{2}$, N. Casasayas-Barris ${ }^{3,4}$, A. Reiners ${ }^{1}$, E. Pallé ${ }^{3,4}$, Th. Henning ${ }^{5}$, P. Mollière ${ }^{5}$, S. Czesla ${ }^{6}$, \\ L. Nortmann ${ }^{1}$, K. Molaverdikhani ${ }^{7}, 5$, G. Chen ${ }^{8}$, I. A. G. Snellen ${ }^{9}$, M. Zechmeister ${ }^{1}$, C. Huang ${ }^{10}$, I. Ribas ${ }^{11,12}$, \\ A. Quirrenbach ${ }^{7}$, J. A. Caballero ${ }^{13}$, P. J. Amado ${ }^{14}$, D. Cont $^{1}$, S. Khalafinejad ${ }^{7}$, J. Khaimova ${ }^{1}$, M. López-Puertas ${ }^{14}$, D. \\ Montes $^{15}$, E. Nagel ${ }^{16}$, M. Oshagh ${ }^{3,4}$, S. Pedraz ${ }^{17}$, and M. Stangret ${ }^{3,4}$
}

${ }^{1}$ Institut für Astrophysik, Georg-August-Universität, Friedrich-Hund-Platz 1, 37077 Göttingen, Germany e-mail: fei.yan@uni-goettingen.de

2 Université Grenoble Alpes, CNRS, IPAG, 38000 Grenoble, France

3 Instituto de Astrofísica de Canarias (IAC), Calle Vía Lactea s/n, 38200 La Laguna, Tenerife, Spain

${ }^{4}$ Departamento de Astrofísica, Universidad de La Laguna, 38026 La Laguna, Tenerife, Spain

5 Max-Planck-Institut für Astronomie, Königstuhl 17, 69117 Heidelberg, Germany

${ }^{6}$ Hamburger Sternwarte, Universität Hamburg, Gojenbergsweg 112, 21029 Hamburg, Germany

7 Landessternwarte, Zentrum für Astronomie der Universität Heidelberg, Königstuhl 12, 69117 Heidelberg, Germany

8 Key Laboratory of Planetary Sciences, Purple Mountain Observatory, Chinese Academy of Sciences, Nanjing 210023, China

${ }^{9}$ Leiden Observatory, Leiden University, Postbus 9513, 2300 RA, Leiden, The Netherlands

${ }^{10}$ Lunar and Planetary Laboratory, 1629 E. University Blvd., P.O. Box 210092, Tucson, AZ 85721-0092, United States of America

11 Institut de Ciències de l'Espai (CSIC-IEEC), Campus UAB, c/ de Can Magrans s/n, 08193 Bellaterra, Barcelona, Spain

12 Institut d'Estudis Espacials de Catalunya (IEEC), 08034 Barcelona, Spain

13 Centro de Astrobiología (CSIC-INTA), ESAC, Camino bajo del castillo s/n, 28692 Villanueva de la Cañada, Madrid, Spain

14 Instituto de Astrofísica de Andalucía (IAA-CSIC), Glorieta de la Astronomía s/n, 18008 Granada, Spain

15 Departamento de Física de la Tierra y Astrofísica and IPARCOS-UCM (Instituto de Física de Partículas y del Cosmos de la UCM), Facultad de Ciencias Físicas, Universidad Complutense de Madrid, E-28040, Madrid, Spain

16 Thüringer Landessternwarte Tautenburg, Sternwarte 5, 07778 Tautenburg, Germany

17 Centro Astronónomico Hispano-Alemán (CSIC-MPG), Observatorio Astronónomico de Calar Alto, Sierra de los Filabres, E-04550 Gérgal, Almería, Spain

Received August 31, 2020; accepted November 15, 2020

\begin{abstract}
Ultra-hot Jupiters (UHJs) are highly irradiated giant exoplanets with extremely high day-side temperatures, which lead to thermal dissociation of most of the molecular species. It is expected that the neutral hydrogen atom is one of the main species in the upper atmospheres of ultra-hot Jupiters. Neutral hydrogen has been detected in several UHJs by observing its Balmer line absorption. Here, we report four transit observations of the ultra-hot Jupiter WASP-33b, performed with the CARMENES and HARPS-North spectrographs, and the detection of the $\mathrm{H} \alpha, \mathrm{H} \beta$, and $\mathrm{H} \gamma$ lines in the planetary transmission spectrum. The combined $\mathrm{H} \alpha$ transmission spectrum of the four transits has an absorption depth of $0.99 \pm 0.05 \%$, which corresponds to an effective radius of $1.31 \pm 0.01 R_{\mathrm{p}}$. The strong $\mathrm{H} \alpha$ absorption indicates that the line probes the high-altitude thermosphere. We further fitted the three Balmer lines using the PAWN model, assuming that the atmosphere is hydrodynamic and in LTE. We retrieved a thermosphere temperature $12200_{-1000}^{+1300} \mathrm{~K}$ and

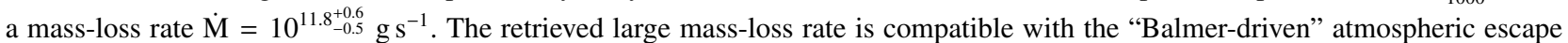
scenario, in which the stellar Balmer continua radiation in the near-ultraviolet is substantially absorbed by the excited hydrogen atoms in the planetary thermosphere.
\end{abstract}

Key words. planets and satellites: atmospheres - techniques: spectroscopic - planets and satellites: individuals: WASP-33b

\section{Introduction}

Ultra-hot Jupiters (UHJs) are the hottest giant exoplanets and they are extensively irradiated by their host stars. These planets are ideal laboratories to study the chemistry and physics of planetary atmospheres under extreme conditions. Theoretical modelling of UHJ atmospheres (e.g., Lothringer et al. 2018; Parmentier et al. 2018; Kitzmann et al. 2018; Helling et al. 2019) suggests that the day-sides as well as the terminators of UHJs are extremely hot and probably dominated by atoms and ions instead of molecules due to thermal dissociation and ionisation.
The thermal emission spectra of several UHJs, including HAT-P-7b (Mansfield et al. 2018), WASP-12b (Stevenson et al. 2014), WASP-18b (Arcangeli et al. 2018), and WASP-103b (Kreidberg et al. 2018), have been observed with the Hubble Space Telescope (HST). These thermal spectra exhibit a lack of $\mathrm{H}_{2} \mathrm{O}$ features, which is probably due to thermal dissociation (Parmentier et al. 2018). On the other hand, emission spectroscopy with high-resolution spectrographs has revealed the existence of neutral Fe in KELT-9 (Pino et al. 2020), WASP-189b (Yan et al. 2020), and WASP-33b (Nugroho et al. 2020a). In ad- 
dition to the thermal emission spectra, phase curve observations have been performed for several UHJs, including WASP-33b (Zhang et al. 2018; von Essen et al. 2020), WASP-121b (Daylan et al. 2019; Bourrier et al. 2020b), and KELT-9b (Wong et al. 2020; Mansfield et al. 2020). These observations suggest that these UHJs have relatively low day-night temperature contrasts and relatively high heat transport efficiencies. The increased heat transport efficiency in UHJs could be explained by a new physical mechanism - thermal dissociation and recombination of $\mathrm{H}_{2}$ (Bell \& Cowan 2018; Komacek \& Tan 2018).

Transmission spectroscopy has also been widely used in probing the atmospheres of UHJs, and various atomic and ionic species have been detected. In the atmosphere of KELT-9b - the hottest exoplanet discovered so far, the hydrogen Balmer lines as well as multiple metal lines (including Fe I, Fe II, Ti II, Mg I, and $\mathrm{Ca}$ II) have been detected (Yan \& Henning 2018; Hoeijmakers et al. 2018; Cauley et al. 2019; Hoeijmakers et al. 2019; Yan et al. 2019; Turner et al. 2020). Various metals as well as the Balmer lines have been detected in KELT-20b/MASCARA-2b (Casasayas-Barris et al. 2018, 2019; Stangret et al. 2020; Nugroho et al. 2020b; Hoeijmakers et al. 2020). Ca II is detected in WASP-33b (Yan et al. 2019). The Balmer lines and metals including $\mathrm{Na}$ I, Mg II, Fe I, Fe II, CrI, and $\mathrm{V}_{\text {I }}$ have been detected in WASP-121b (Sing et al. 2019; Bourrier et al. 2020a; Gibson et al. 2020; Cabot et al. 2020; Ben-Yami et al. 2020). $\mathrm{H} \alpha$ and Mg II have been discovered in WASP-12b (Fossati et al. 2010; Jensen et al. 2018). Neutral Fe has also been detected at the terminator of WASP-76b (Ehrenreich et al. 2020).

Planets experiencing strong stellar irradiation are thought to undergo hydrodynamic atmospheric escape (e.g., Owen 2019, and references therein). The hydrodynamic escape in hydrogendominated atmospheres is normally driven by the absorption of stellar extreme-ultraviolet (EUV) flux (Yelle 2004; Tian et al. 2005; Salz et al. 2016). However, Fossati et al. (2018) found that heating due to atomic absorption of the stellar UV and optical flux drives the atmospheric escape of UHJs orbiting early-type stars. García Muñoz \& Schneider (2019) further proposed that the absorption of the hydrogen Balmer line series can enhance and even drive the atmospheric escape of UHJs orbiting hot stars.

Observations of atmospheric escape have been performed with the hydrogen Ly $\alpha$ line (Vidal-Madjar et al. 2003; Lecavelier des Etangs et al. 2012; Ehrenreich et al. 2015) as well as metal lines in the ultraviolet (Fossati et al. 2010; Sing et al. 2019; $\mathrm{Cu}-$ billos et al. 2020), using the STIS spectrograph on HST. The helium $10833 \AA$ line has recently been used in probing escaping atmosphere of planets orbiting active stars (e.g., Spake et al. 2018; Nortmann et al. 2018; Allart et al. 2018; Salz et al. 2018; Lampón et al. 2020; Palle et al. 2020).

The hydrogen Balmer lines can also be used to probe highaltitude atmospheres and study atmospheric escape. For example, Yan \& Henning (2018) estimated the Jeans escape rate of KELT-9b with the $\mathrm{H} \alpha$ absorption line. Recently, Wyttenbach et al. (2020) modelled the Balmer lines with a hydrodynamic model and retrieved the mass-loss rate of KELT-9b. The Balmer lines have been detected in four UHJs so far: KELT-9b (Yan \& Henning 2018; Cauley et al. 2019; Turner et al. 2020), KELT-20b (Casasayas-Barris et al. 2018, 2019), WASP-12b (Jensen et al. 2018), and WASP-121b (Cabot et al. 2020). Besides, the $\mathrm{H} \alpha$ line has been detected in two non-UHJ planets - HD $189733 \mathrm{~b}$ (Jensen et al. 2012; Barnes et al. 2016; Cauley et al. 2016, 2017a,b) and WASP-52b (Chen et al. 2020). The $\mathrm{H} \alpha$ absorption in these two planets probably originates from the excitation of hydrogen atoms due to stellar Ly $\alpha$ line and Lyman continuum irradiation (Christie et al. 2013; Huang et al. 2017).
Here, we present the discovery of the Balmer line absorption during the transit of WASP-33b - a UHJ (equilibrium temperature $\sim 2710 \mathrm{~K}$ ) orbiting an A5 star (Collier Cameron et al. 2010). Several species have previously been detected in its planetary atmosphere, including TiO (Haynes et al. 2015; Nugroho et al. 2017), Ca II (Yan et al. 2019), Fe I (Nugroho et al. 2020a), and evidence of AlO (von Essen et al. 2019) and FeH (Kesseli et al. 2020). The paper is organised as follows. We describe the transit observations and data analysis in Sect. 2. The observational results are presented in Sect. 3. In Sect. 4, we present the hydrodynamic model of the Balmer lines and discuss the atmospheric escape of WASP-33b. The conclusions are summarised in Sect. 5.

\section{Data and analysis}

\subsection{Observations}

We observed four transits of WASP-33b with two spectrographs. The observation logs are summarised in Table 1.

Two transits were observed with the CARMENES (Quirrenbach et al. 2018) installed at the $3.5 \mathrm{~m}$ telescope of the Calar Alto Observatory on 5 January 2017 and 16 January 2017. The visual channel of the CARMENES spectrograph has a resolution of $R \sim 94600$ and a wavelength coverage of 520-960 nm. The first night was photometric (i.e., ideal weather condition during the observation) and the second night was partially cloudy.

Another two transits were observed with the HARPS-North (HARPS-N) spectrograph mounted on the Telescopio Nazionale Galileo telescope on 17 October 2018 and 8 November 2018. The instrument has a resolution of $R \sim 115000$ and a wavelength coverage of 383-690 nm. We used the order-merged onedimensional spectra from the HARPS-N pipeline (Data Reduction Software). The spectra have an over-sampled wavelength step of $0.01 \AA$. We re-binned the spectrum every 3 wavelength points by averaging so that each wavelength point corresponds to $0.03 \AA$, which is similar to the CARMENES pixel size at the $\mathrm{H} \alpha$ line centre $(0.030 \AA)$. Both nights were photometric. However, the spectral flux from the first night observation had a large drop when the telescope was pointing close to the zenith. Such a phenomenon also occurred during transit observations in CasasayasBarris et al. (2019), which was probably caused by a problem with the atmospheric dispersion corrector (ADC).

The signal-to-noise ratio $(\mathrm{S} / \mathrm{N})$ per wavelength point $(\sim 0.03$ $\AA$ ) at the $\mathrm{H} \alpha$ line centre is plotted in Fig.1. Among the four transits, night-1 from CARMENES and night-2 from HARPS-N observations have much higher $\mathrm{S} / \mathrm{N}$ and, therefore, were used in Yan et al. (2019) for the detection of ionised calcium. In this work, we use and combine all four transits.

\subsection{Obtaining the transmission spectral matrix}

We investigated the $\mathrm{H} \alpha$ line (6562.79 $\AA$ ) using both the CARMENES and HARPS-N observations and the $\mathrm{H} \beta$ $(4861.35 \AA)$ and $\mathrm{H} \gamma(4340.47 \AA)$ lines from the HARPS-N observations. The data reduction method is similar to the method in Yan \& Henning (2018).

The spectra were first normalised and shifted into the Earth's rest frame. We then removed the telluric absorption lines using a theoretical transmission spectral model of the Earth's atmosphere (Yan et al. 2015b). The spectra were subsequently aligned 
Table 1. Observation logs.

\begin{tabular}{lcccccc}
\hline \hline Instrument & & Date & Observing Time (UT) & Airmass change & Exposure time [s] & $N_{\text {spectra }}$ \\
\hline CARMENES & Night-1 & $2017-01-05$ & $19: 28-23: 49$ & $1.00-1.54$ & $120^{a}$ & 93 \\
CARMENES & Night-2 & $2017-01-16$ & $19: 25-00: 07$ & $1.01-2.03$ & 120 & 66 \\
\hline HARPS-N & Night-1 & $2018-10-17$ & $21: 39-05: 46$ & $1.64-1.01-1.54$ & 200 & 124 \\
HARPS-N & Night-2 & $2018-11-08$ & $19: 59-05: 01$ & $1.74-1.01-1.87$ & 200 & 141 \\
\hline
\end{tabular}

Notes. ${ }^{(a)}$ The first 19 spectra had exposure time below $120 \mathrm{~s}$.

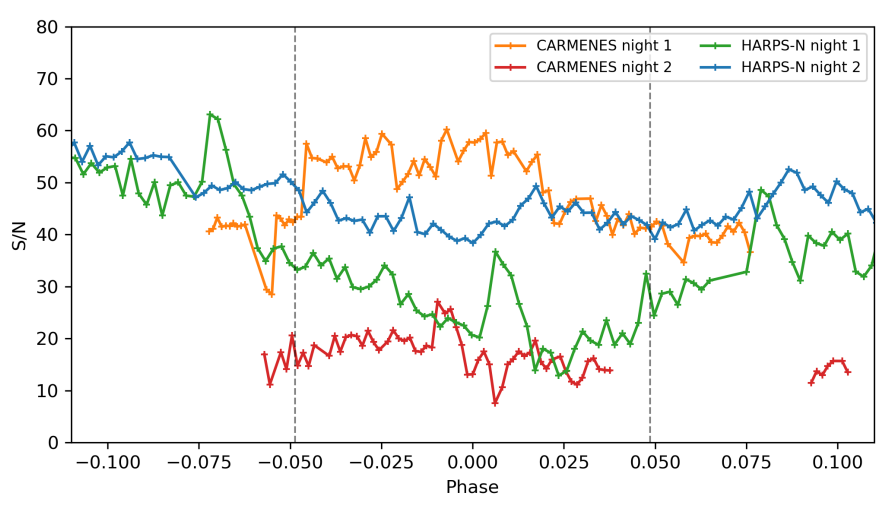

Fig. 1. Signal-to-noise ratio per wavelength point $(\sim 0.03 \AA)$ at the $\mathrm{H} \alpha$ line centre. The dashed lines indicate the beginning and end of transit.

into the stellar rest frame by correcting the barycentric radial velocity and the stellar systemic velocity $\left(-3.0 \mathrm{~km} \mathrm{~s}^{-1}\right.$, Nugroho et al. 2017). We obtained an out-of-transit master spectrum by adding up all the out-of-transit spectra with the squared $\mathrm{S} / \mathrm{N}$ as weight. We then divided each spectrum by the master spectrum in order to remove the stellar lines. The residual spectrum was subsequently filtered with a Gaussian high-pass filter $(\sigma \sim 300$ points) to remove large scale features on the continuum spectrum, which may be attributed to the stability of the HARPS$\mathrm{N}$ ADC, stellar pulsation, or the imperfect normalisation of the blaze variation.

We combined the two CARMENES observations as well as the two HARPS-N observations by binning the spectra with an orbital phase step of 0.005 . The binning was performed by averaging the spectra within each phase bin with the squared $\mathrm{S} / \mathrm{N}$ as weight.

By applying these procedures, we obtained a transmission spectral matrix for each of the $\mathrm{H} \alpha, \mathrm{H} \beta$, and $\mathrm{H} \gamma$ lines from the HARPS-N observations and an $\mathrm{H} \alpha$ spectral matrix from the CARMENES observations (upper panels in Figs. 2 and 3).

\subsection{Model of stellar RM and CLV effects}

The stellar line profile varies during the transit due to the Rossiter-McLaughlin (RM) effect (Queloz et al. 2000) and the centre-to-limb variation (CLV) effect (Yan et al. 2015a; Czesla et al. 2015; Yan et al. 2017). We modelled the RM and CLV effects simultaneously following the method described in Yan \& Henning (2018). We used the same stellar and planetary parameters as in Yan et al. (2019).

The planetary orbit of WASP-33b undergoes a nodal precession (Johnson et al. 2015; Iorio 2016; Watanabe et al. 2020). We adopted the orbital change rates from Johnson et al. (2015) and calculated the expected orbital inclination $(i)$ and spin-orbit an- gle $(\lambda)$ at the dates of our observations. Because the observation dates are very close for the two CARMENES transits as well as for the two HARPS-N transits, the changes of the orbital parameters between them are negligible. Therefore, we set $i=89.50$ deg and $\lambda=-114.05 \mathrm{deg}$ for the combined CARMENES transmission matrix; $i=90.14 \mathrm{deg}$ and $\lambda=-114.93 \mathrm{deg}$ for the combined HARPS-N transmission matrix.

\subsection{Fitting the observed spectral matrix}

We fitted the observed transmission spectral matrix with a model consisting of two components: the planetary absorption and the stellar line profile change. We assumed that the planetary absorption has a Gaussian profile described by the full width at half maximum (FWHM), the absorption depth $(h)$, and the radial velocity (RV) shift of the observed line centre compared to the theoretical value $\left(V_{\text {centre }}\right)$. The semi-amplitude of the planetary orbital motion $\left(K_{\mathrm{p}}\right)$ is fixed to the expected $K_{\mathrm{p}}$ value $(231 \pm 3$ $\mathrm{km} \mathrm{s}^{-1}$ ), which is calculated with the planetary orbital parameters. The stellar line profile change caused by the RM and CLV effects is fixed to the results as calculated in Sect. 2.3.

We sampled from the posterior probability distribution using the Markov Chain Monte Carlo (MCMC) simulations with the emcee tool (Foreman-Mackey et al. 2013). We only used the fully in-transit data (i.e. excluding the ingress and egress phases).

\section{Results and discussion}

\section{1. $\mathrm{H} \alpha$ transmission spectrum}

The transmission spectral matrices are shown in Fig. 2a. The $\mathrm{H} \alpha$ absorption is clearly detected in both CARMENES and HARPS$\mathrm{N}$ data. The best-fit models of the planetary absorption feature as well as the stellar CLV and RM effects are shown in Fig. 2b, and the best-fit parameters are summarised in Table 2 . In order to obtain the one-dimensional transmission spectra, we firstly corrected the CLV and RM effects. Then, the residual spectra were shifted into the planetary rest frame. We subsequently averaged all the fully in-transit spectra to derive the final one-dimensional transmission spectra, which are presented in Fig. 4. The $\mathrm{H} \alpha$ transmission spectrum of each night is shown in Fig. A.1.

The obtained FWHM is $31.6_{-3.6}^{+4.1} \mathrm{~km} \mathrm{~s}^{-1}$ for HARPS-N and $35.6_{-2.0}^{+2.2} \mathrm{~km} \mathrm{~s}^{-1}$ for CARMENES. These values are smaller than those of KELT-9b (Yan \& Henning 2018; Cauley et al. 2019; Turner et al. 2020) while slightly higher than those of KELT-20b (Casasayas-Barris et al. 2018, 2019). The large FWHM indicates that the $\mathrm{H} \alpha$ absorption is optically thick (Huang et al. 2017).

The measured RV shift of the line centre is $2.0 \pm 1.9 \mathrm{~km} \mathrm{~s}^{-1}$ for HARPS-N and $0.8 \pm 1.1 \mathrm{~km} \mathrm{~s}^{-1}$ for CARMENES. The RV shift has been used to measure high-altitude winds at the planetary terminator (Snellen et al. 2010; Wyttenbach et al. 2015; Louden \& Wheatley 2015; Brogi et al. 2016). Nevertheless, the 

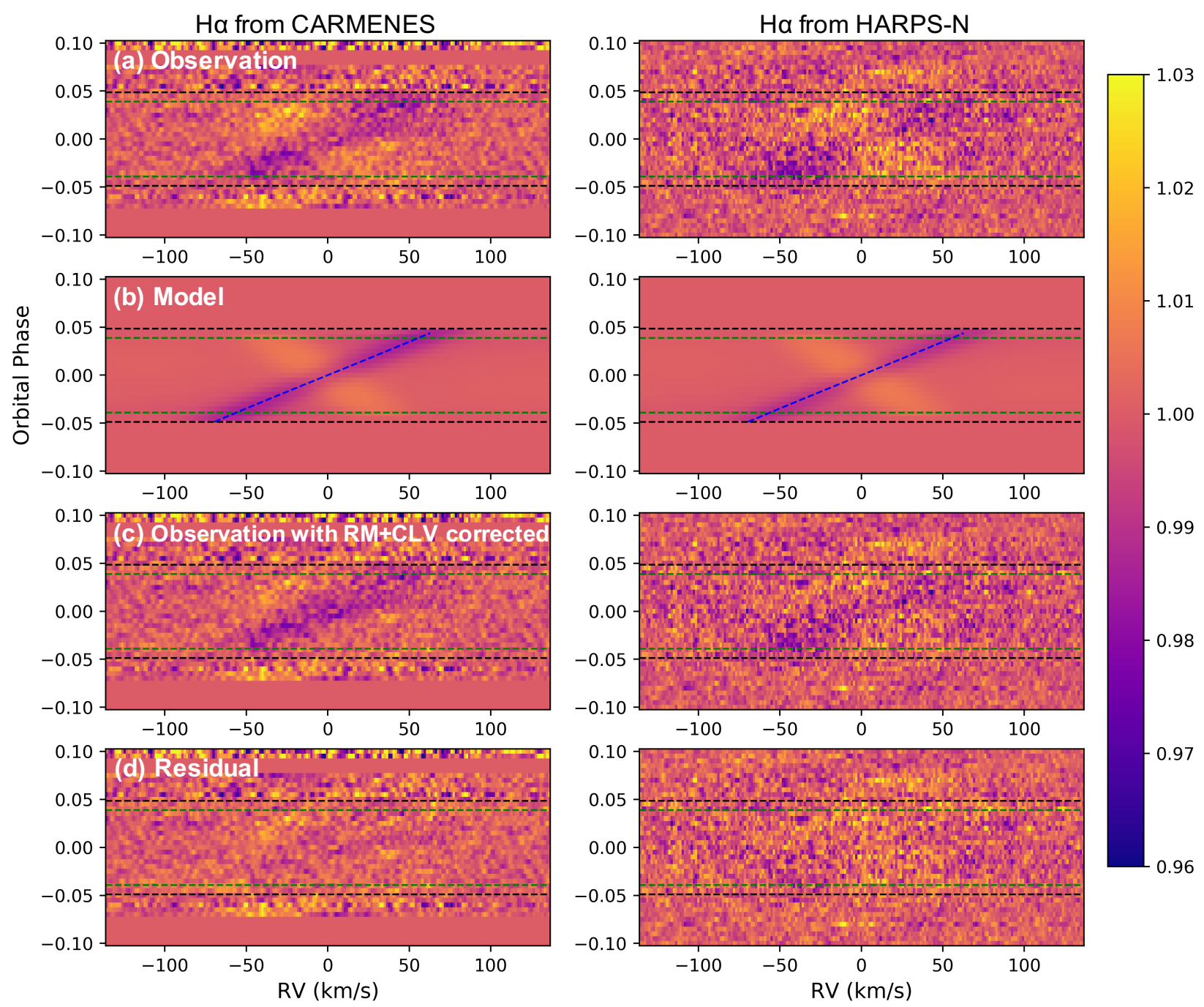

Fig. 2. Transmission spectral matrices for the $\mathrm{H} \alpha$ line from the CARMENES observations (left) and the HARPS-N observations (right). The color bar indicates the value of relative flux. (a) The observed transmission spectra. The $\mathrm{x}$ axis is wavelength expressed in RV relatively to the H $\alpha$ line centre $(6562.79 \AA)$ in the stellar rest frame. The horizontal dashed lines indicate the four contacts of transit. (b) The best-fit model from the MCMC analysis. The model includes the $\mathrm{H} \alpha$ transmission spectrum and the stellar line profile change (i.e. the CLV and RM effects). The blue dashed line indicates the RV of the planetary orbital motion plus a constant shift $\left(V_{\text {centre }}\right)$. Although the models extend into the ingress and egress regions on the matrices, the fit was only performed on the fully in-transit data. (c) The observed transmission spectra with the RM and CLV effects corrected. (d) The residual between the observation and the model.

measured $V_{\text {centre }}$ is relative to the stellar systemic RV and there is a large uncertainty of the stellar RV of WASP-33. This is because precisely measuring the absolute RV of fast-rotating A-type stars is intrinsically challenging. For example, the reported systemic RVs of WASP-33 deviate by several $\mathrm{km} \mathrm{s}^{-1}$ (Collier Cameron et al. 2010; Lehmann et al. 2015; Nugroho et al. 2017; Cauley et al. 2020b). Therefore, we conclude that we do not detect any significant winds at the terminator of WASP-33b considering the uncertainties in the measured $V_{\text {centre }}$ values and the stellar systemic RV.

We further combined the CARMENES and HARPS-N transmission spectral matrices using the binning method as described in Section 2.2. The stellar CLV and RM effects were already corrected before the averaging. The combined matrix is presented in Fig. 5 and the best-fit parameters are listed in Table 2. We calculated the equivalent width of the absorption line $\left(W_{\mathrm{H} \alpha}\right)$ using the same method as in Yan \& Henning (2018), except that the integration range was set as $\pm 35 \mathrm{~km} \mathrm{~s}^{-1}$ to match the observed FWHM. Fig. 6 shows the time series of $W_{\mathrm{H} \alpha}$. There is no obvious pre- or after-transit absorption, although the absorption depth is slightly stronger during the first-half transit.

In general, the fitted parameters between CARMENES and HARPS-N are consistent. However, the CARMENES H $\alpha$ absorption is somewhat stronger than the HARPS-N absorption. Such a slight difference between the two instruments is also observed for the $\mathrm{H} \alpha$ line in KELT-20b (Casasayas-Barris et al. 2019) and KELT-9b (Yan \& Henning 2018; Wyttenbach et al. 2020; Turner et al. 2020). Although the difference could be from random variations, there may also be systematic residuals, which could be due to instrumental effects (e.g., non-linearity, the stability of the HARPS-N ADC) or data reduction procedures (e.g., imperfect normalisation, removal of stellar and telluric lines).

For the case of WASP-33b, the slight difference between the CARMENES and HARPS-N results could be caused by the stel- 

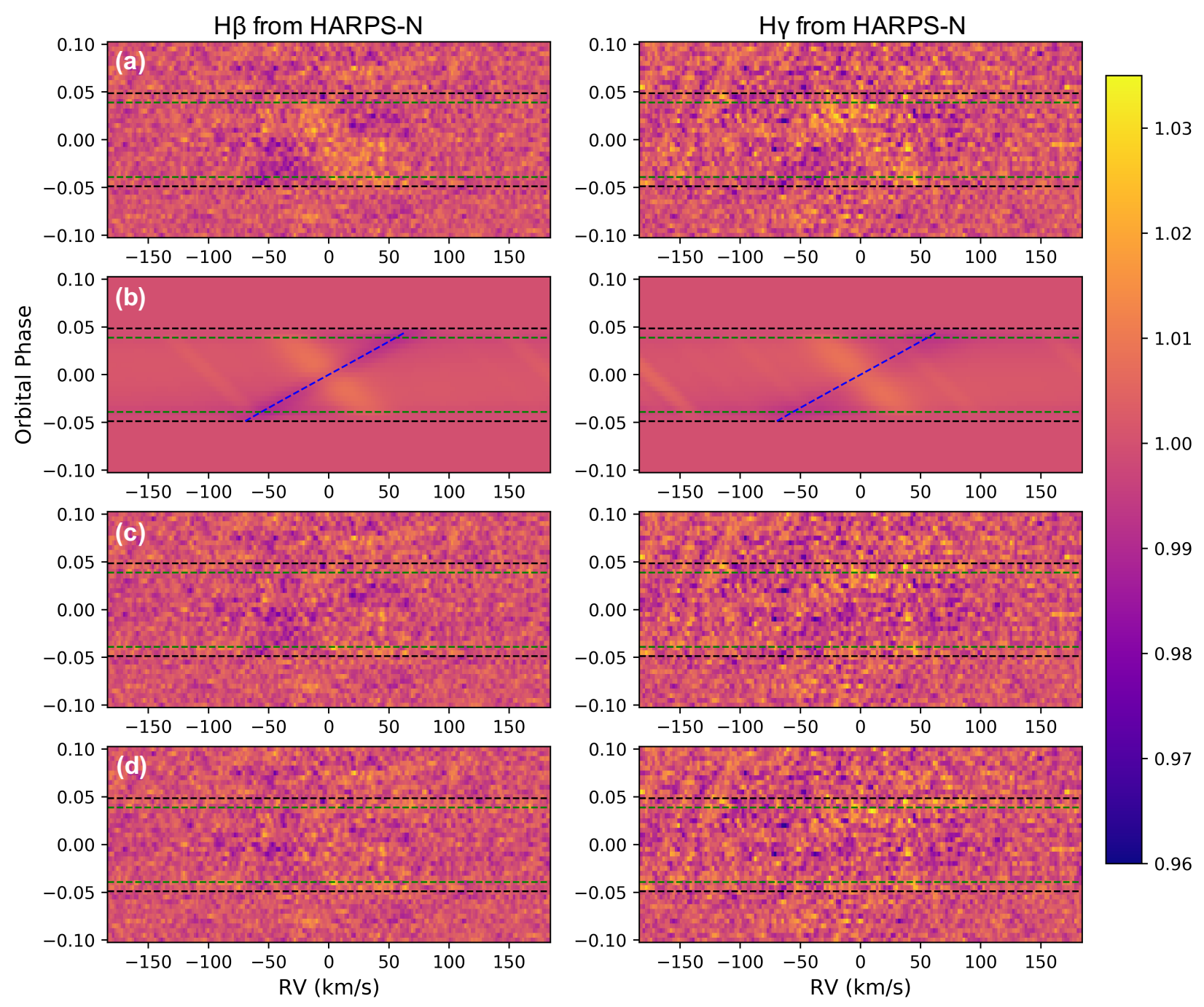

Fig. 3. Same as Fig. 2 but for the $\mathrm{H} \beta$ line (left) and the $\mathrm{H} \gamma$ line (right) from the HARPS-N observations.

lar pulsations, which can affect the stellar $\mathrm{H} \alpha$ line profile. The host star is a known $\delta$ Scuti star with pronounced pulsations (Collier Cameron et al. 2010; von Essen et al. 2014; Kovács et al. 2013). For the CARMENES spectrum in Fig. 4, there is a bump feature on the left of the planetary absorption line. A weaker bump feature is also present in the HARPS-N spectrum. Such a bump feature is also observed in the transmission spectrum of the Ca II infrared triplet lines (Yan et al. 2019), which are obtained using the same transit data as in this work. On the combined transmission spectral matrix in Fig. 5, there are bright stripes on the left and right sides of the planetary absorption signal and these stripes extend beyond the transit. These stripes are probably the stellar pulsation signatures, which generate the bump features as observed on the transmission spectra in Fig. 4. For individual CARMENES and HARPS-N observation, the position and strength of the pulsation features were not the same during the transits. Therefore, the stellar pulsation could introduce a difference between the CARMENES and HARPS-N results. In a preprint posted while the present paper was under review, Cauley et al. (2020b) reported the detection of the Balmer lines in WASP-33b using the PEPSI spectrograph mounted on the Large Binocular Telescope. Their obtained absorption line strength is quantitatively different but generally consistent with our CARMENES and HARPS-N results, considering possible effects of the stellar pulsation.

Although the detection of the $\mathrm{H} \alpha$ line is unambiguous, its strength is potentially affected by the stellar pulsation. To correct the effect of the pulsation, a detailed analysis of the variation in the stellar Balmer lines is required. Such an analysis requires data taken with high $\mathrm{S} / \mathrm{N}$ and is beyond the scope of this paper. However, considering that the pulsating periods are not synchronous with the planetary orbital motion (von Essen et al. 2014), the pulsating contribution to the transmission spectrum should be statistically reduced when combining the four transit spectra together. To evaluate the effect of pulsation, we combined the out-of-transit spectra on the spectral matrix in Fig.5 (i.e., phases -0.10 to -0.05 and +0.05 to +0.10 ) and assumed the in-transit orbital velocity to repeat during out-of-transit. The obtained out-of-transit spectrum (Fig.7) shows ripple-like features with semi-amplitude of $\sim 0.2 \%$, which are most likely the results of the stellar pulsation. The effect of the pulsation to the $\mathrm{H} \alpha$ transmission spectrum should be at a similar order of these ripple features. 


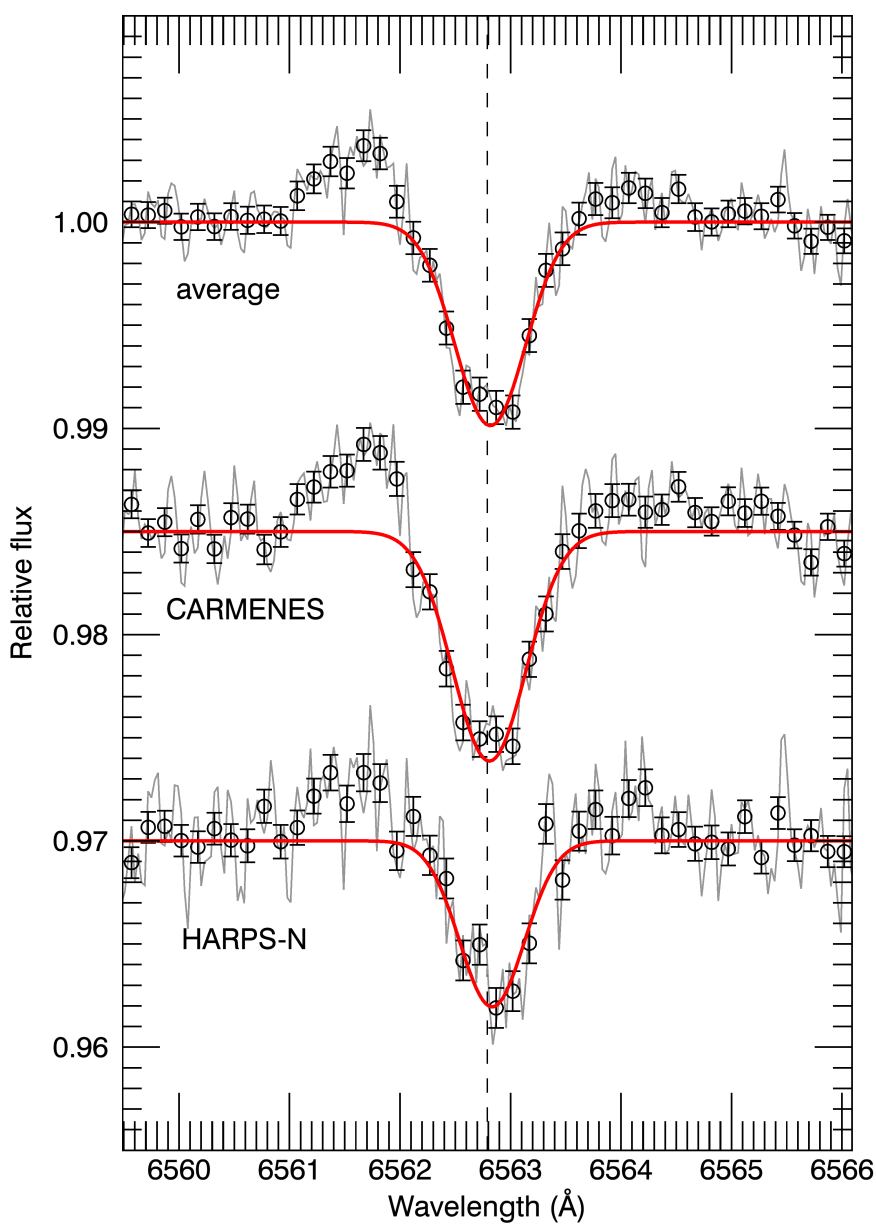

Fig. 4. Transmission spectra of the $\mathrm{H} \alpha$ line. The black circles are spectra binned every five points $(\sim 0.15 \AA)$ and the grey lines are the original spectra (i.e., $\sim 0.03 \AA$ per point). The red lines are the best-fit Gaussian functions. The vertical dashed line indicates the rest wavelength line centre. The CLV and RM effects are corrected. An offset of the y-axis is applied to the spectra for clarity.

We note that Valyavin et al. (2018) analysed the transit light curve of WASP-33b observed with the $\mathrm{H} \alpha$ filter. These latter authors found that the $\mathrm{H} \alpha$ transit depth is significantly deeper than the transit depths measured in broad bands. Since we detect the strong $\mathrm{H} \alpha$ absorption line with high-resolution spectroscopy, we confirm that the photometric result of Valyavin et al. (2018) is evidence of the $\mathrm{H} \alpha$ absorption in the planetary atmosphere.

\section{2. $\mathrm{H} \beta$ and $\mathrm{H} \gamma$ transmission spectrum}

The $\mathrm{H} \beta$ and $\mathrm{H} \gamma$ lines are only covered by the HARPS-N spectrograph. The absorption signals are relatively weak compared to the $\mathrm{H} \alpha$ line. The best-fit parameters are shown in Table 2 with the final transmission spectra in Fig. 8. The detection of $\mathrm{H} \beta$ is clear while the $\mathrm{H} \gamma$ signal is less prominent. The line depth of the $\mathrm{H} \beta$ absorption is smaller than that of the $\mathrm{H} \alpha$ line, but their FWHM values are relatively similar to each other. This is also the case for the $\mathrm{H} \alpha$ and $\mathrm{H} \beta$ lines in KELT-9b (Cauley et al. 2019; Wyttenbach et al. 2020) and KELT-20b (Casasayas-Barris et al. 2019).

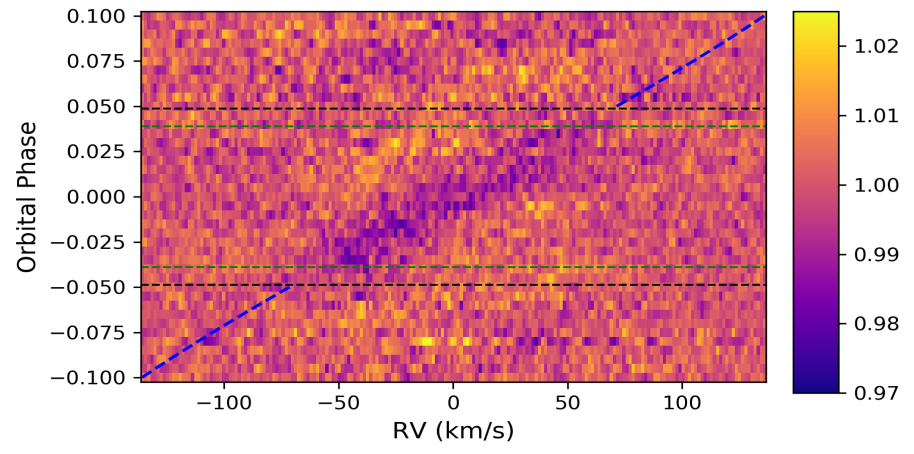

Fig. 5. Combined transmission spectral matrix of the CARMENES and HARPS-N results for the $\mathrm{H} \alpha$ line. The stellar line profile change due to the CLV and RM effects has been removed before averaging. The horizontal dashed lines indicate the four contacts of transit and the diagonal dashed lines denote the planetary orbital RV.

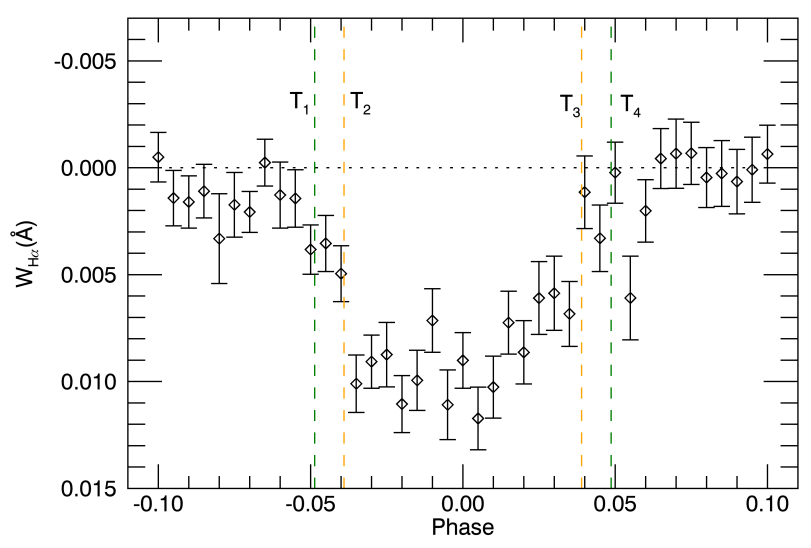

Fig. 6. Time series of the $\mathrm{H} \alpha$ equivalent width. The values are measured on the combined transmission spectral matrix in Fig. 5. The vertical dashed lines indicate the first $\left(\mathrm{T}_{1}\right)$, second $\left(\mathrm{T}_{2}\right)$, third $\left(\mathrm{T}_{3}\right)$, and fourth $\left(\mathrm{T}_{4}\right)$ contacts of the transit. The horizontal line denotes $W_{\mathrm{H} \alpha}=0$.

\subsection{Model of the Balmer lines}

\subsubsection{Estimation of the atmospheric conditions}

In order to obtain a rough estimate of the possible atmospheric conditions, and before modelling the lines, one can use the Lecavelier Des Etangs et al. (2008) formula to estimate the atmospheric scale-height in the region probed by the Balmer lines. Indeed, the altitude of absorption $z$ is proportional to the hydrogen Balmer line oscillator strengths $\ln (g f): \Delta z=H \Delta \ln (g f)$, where $H=k_{B} T / \mu g$ is the pressure scale-height. The $\ln (g f)$ values are $1.635,-0.046$, and -1.029 for the $\mathrm{H} \alpha, \mathrm{H} \beta$, and $\mathrm{H} \gamma$ lines, respectively. Taking into account all our measurements from CARMENES and HARPS-N, we computed $H=9200 \pm$ $1200 \mathrm{~km}$. Considering the decrease of the gravity $g$ with altitude, we estimated that $T / \mu \simeq 15100 \pm 2100[\mathrm{~K} / \mathrm{u}]\left(\right.$ at $\left.z \sim 1.2 \mathrm{R}_{\mathrm{P}}\right)$. As it is likely that molecular hydrogen is dissociated under these conditions, we can further assume that $\mu$ is between 0.66 and 1.26 (the atmosphere is dominated by a mixture of ionised and neutral hydrogen and helium). Hence, we estimated the upper atmosphere temperature to be between 8600 and $21700 \mathrm{~K}$. The lower end of the $T$ range should be preferred since when the tem- 
Table 2. Fit results of the transmission spectral matrices.

\begin{tabular}{llccccc}
\hline \hline & & $V_{\text {centre }}\left[\mathrm{km} \mathrm{s}^{-1}\right]$ & FWHM $\left[\mathrm{km} \mathrm{s}^{-1}\right]$ & Line depth [\%] & $R_{\text {eff }}\left[R_{\mathrm{p}}\right]$ & Detection significance \\
\hline & CARMENES & $0.8 \pm 1.1$ & $35.6_{-2.0}^{+2.2}$ & $1.11 \pm 0.07$ & $1.34 \pm 0.02$ & $16 \sigma$ \\
$\mathrm{H} \alpha$ & HARPS-N & $2.0 \pm 1.9$ & $31.6_{-3.6}^{+4.1}$ & $0.81 \pm 0.09$ & $1.26 \pm 0.03$ & $9 \sigma$ \\
& combination & $1.2 \pm 0.9$ & $34.3 \pm 1.6$ & $0.99 \pm 0.05$ & $1.31 \pm 0.01$ & $20 \sigma$ \\
\hline $\mathrm{H} \beta$ & HARPS-N & $2.2 \pm 1.7$ & $30.6_{-4.3}^{+4.9}$ & $0.54 \pm 0.07$ & $1.18 \pm 0.02$ & $8 \sigma$ \\
\hline $\mathrm{H} \gamma$ & HARPS-N & $7 \pm 15$ & $49_{-26}^{+25}$ & $0.28_{-0.15}^{+0.09}$ & $1.10_{-0.05}^{+0.03}$ & $2.3 \sigma$ \\
\hline
\end{tabular}

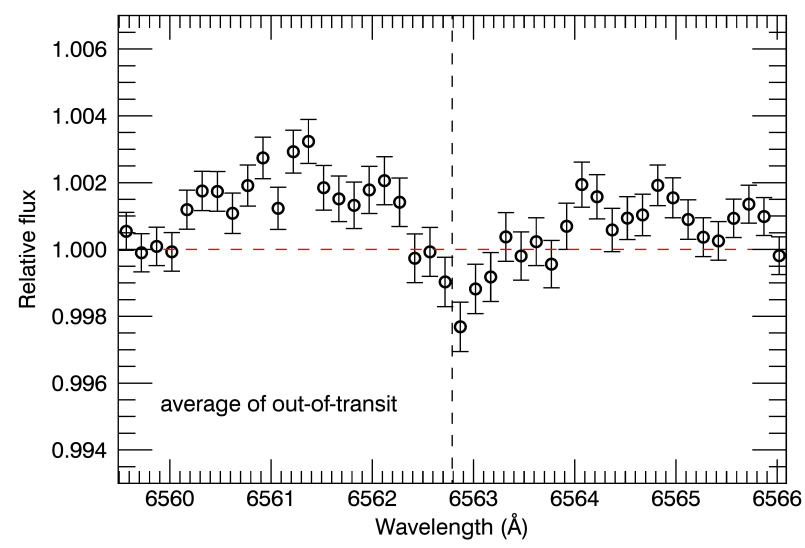

Fig. 7. Average out-of-transit spectrum of the $\mathrm{H} \alpha$ transmission spectral matrix in Fig.5. The vertical dashed line denotes the line centre. These spectral features likely originate from the stellar pulsation.

perature increases, the amount of ionised hydrogen increases, making $\mu$ decreases as well.

\subsubsection{Model set-up}

To interpret the observed Balmer lines in WASP-33b, we employed the PAWN model (PArker Winds and Saha-BoltzmanN atmospheric model) developed by Wyttenbach et al. (2020). This tool is a 1-D model of an exoplanet upper atmosphere linked to an MCMC retrieval algorithm. Its purpose is to retrieve parameters of the thermosphere regions (e.g., the temperature, massloss rate) from high-resolution transmission spectra. Key features of the PAWN model are summarised here. First, we can choose the atmospheric structure to be hydrostatic (barometric law) or hydrodynamic (Parker wind transonic solution), with the base density or the mass-loss rate being a free parameter, respectively. The atmospheric profile is assumed to be isothermal in both cases, with the temperature being an additional free parameter. We also assume the atmosphere to be in chemical equilibrium, with Solar abundances. We use a chemical grid calculated with the equilibrium chemistry code presented in Mollière et al. (2017), from which we interpolate the volume mixing ratios and other useful quantities according to the atmospheric structure. As we detected Balmer lines, we focus on the neutral hydrogen. In local thermodynamic equilibrium (LTE), the number densities of the different electronic states follow the Boltzmann distribution. Then, the opacities have a Voigt profile and follow the prescriptions of Kurucz (1979, 1992); Sharp \& Burrows (2007). Finally, the transmission spectrum is computed following Mollière et al. (2019). The line profiles are broadened taking into account the planetary rotation (tidally locked solid body rotation perpendic-
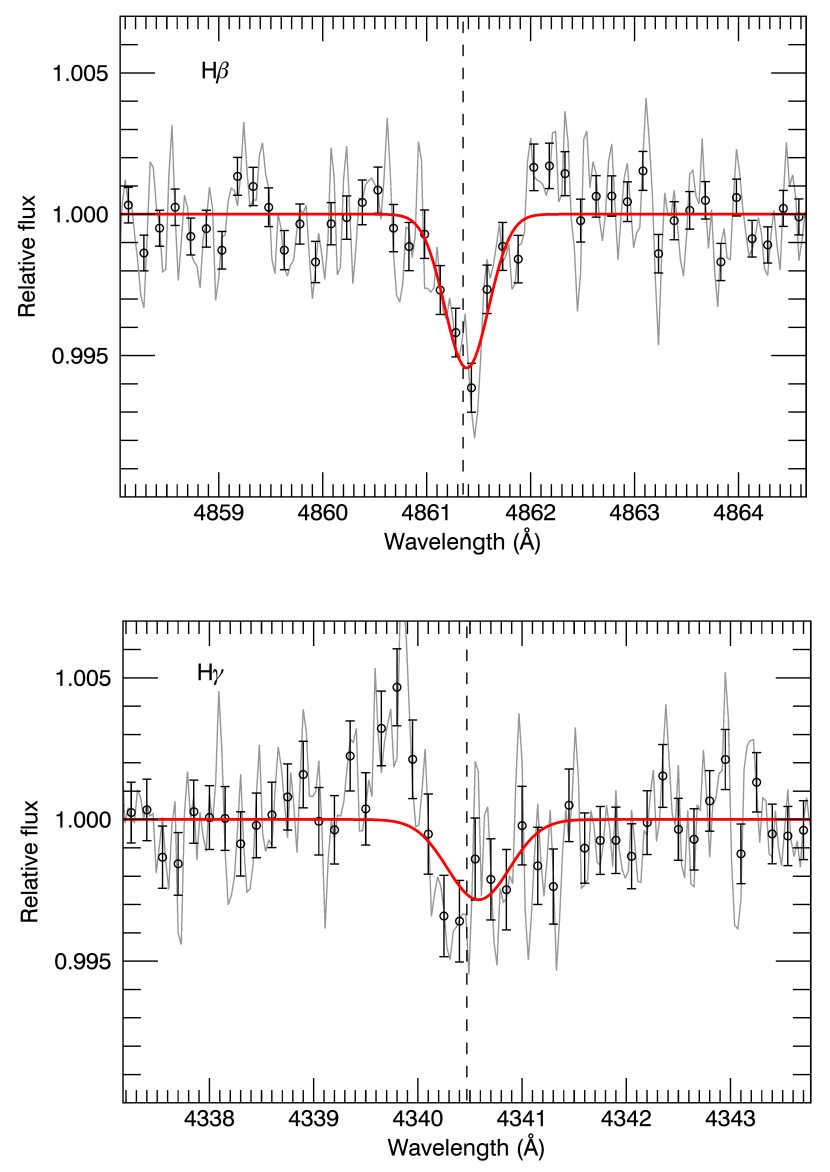

Fig. 8. Transmission spectra of the $\mathrm{H} \beta$ line (upper panel) and the $\mathrm{H} \gamma$ line (lower panel).

ular to the orbital plane). The model is also convolved, binned and normalised in order to be comparable to the data.

On top of the atmospheric model parameters presented above, each line centre is a free parameter. For other planetary parameters (e.g., mass and radius), we used the same values as presented in Table 2 of Yan et al. (2019). For every MCMC chain, we used 10 walkers for each parameter during 2500 steps, with a burn-in size of 500 steps. For each parameter, we used a uniform or log-uniform prior. For the mass-loss rate we put a lower boundary for the prior at $\log _{10}\left(\dot{\mathrm{M}}\left[\mathrm{g} \mathrm{s}^{-1}\right]\right)=9$, as it is expected that WASP-33b is undergoing strong atmospheric escape (Fossati et al. 2018). We tried to fit hydrostatic and hydrodynamic structures to see if one structure would be preferred. The Bayesian information criterion (BIC) allows us to compare the results of different models, and to choose the best-fitting model. 
Table 3. MCMC results of the PAWN modeling for an atmosphere in hydrodynamic expansion and in LTE.

\begin{tabular}{llcc}
\hline \hline & & $T\left[10^{3} \mathrm{~K}\right]$ & $\log _{10}\left(\dot{\mathrm{M}}\left[\mathrm{g} \mathrm{s}^{-1}\right]\right)$ \\
\hline $\mathrm{H} \alpha$ & CARMENES & $14.6_{-2.1}^{+2.4}$ & $12.8_{-0.8}^{+0.6}$ \\
\hline $\mathrm{H} \alpha$ & HARPS-N & $12.6_{-2.6}^{+4.0}$ & $11.8_{-1.4}^{+1.3}$ \\
$\mathrm{H} \beta$ & HARPS-N & $12.8_{-3.3}^{+3.8}$ & $12.1_{-2.0}^{+1.3}$ \\
$\mathrm{H} \gamma$ & HARPS-N & $12.7_{-3.2}^{+4.3}$ & $12.0_{-1.9}^{+1.6}$ \\
\hline $\mathrm{All}$ & Combination & $12.2_{-1.0}^{+1.3}$ & $11.8_{-0.5}^{+0.6}$ \\
\hline
\end{tabular}

\subsubsection{Model results}

We performed MCMC chains on each individual Balmer absorption line from HARPS-N and CARMENES. We also fitted the three Balmer lines simultaneously, using the combined HARPS$\mathrm{N}$ and CARMENES result. It is important to note that since the depth of the $\mathrm{H} \alpha$ line is not the same for the two instruments, we would expect some differences in the retrieved parameters.

The results from the MCMC model fitting are summarised in Table 3 for the case of a hydrodynamic atmosphere in LTE. For each detection, the retrieved parameters are compatible. The combined fit (all Balmer lines from HARPS-N and CARMENES) points toward a thermospheric temperature of $T=12200_{-1000}^{+1300} \mathrm{~K}$ and a mass-loss rate of $\dot{M}=10^{11.8_{-0.5}^{+0.6}} \mathrm{~g} \mathrm{~s}^{-1}$. The best-fit spectra and the correlation diagram of the combined fit are presented in Fig. 9 and Fig. 10, respectively. Before interpreting this result, we mention here that for each scenario (line or instrument), the absorption line was fitted equally well by a hydrostatic structure $(\triangle \mathrm{BIC}<1)$. This is because, for a hot Jupiter, it is often possible to find a very similar atmospheric structure for both cases, especially when the temperature is high (Wyttenbach et al. 2020). Nevertheless, an evaporating scenario could be preferred for WASP-33b as suggested by forward modelling (Fossati et al. 2018). This latter study predicted a mass-loss rate of about $10^{11} \mathrm{~g} \mathrm{~s}^{-1}$ for WASP-33b, which is well in line with our retrieved mass-loss rate.

Our retrieved mass-loss rate of $\dot{M}=10^{11.8_{-0.5}^{+0.6}} \mathrm{~g} \mathrm{~s}^{-1}$ is close to the maximum energy-limited mass-loss rate (Fossati et al. 2018). This could suggest that the heating efficiency is high (on the order of $10-100 \%)$, meaning that most of the irradiation energy goes into expansion ( $P d V$ work) and escape. However, according to Salz et al. (2016), when a hot Jupiter has a relatively high gravitational potential (such as WASP-33b), the heating efficiency should decrease by several orders of magnitude. This hints that the energy-limited computation of Fossati et al. (2018) may not be complete and that some energy sources are not taken into consideration. Indeed, García Muñoz \& Schneider (2019) suggested that hot Jupiters orbiting early type stars could undergo a "Balmer-driven" evaporation. This mechanism has been proposed for the ultra-hot Jupiter KELT-9b and is supported by the observations of the Balmer series in its thermosphere (Yan \& Henning 2018; Wyttenbach et al. 2020). This "Balmer-driven" mechanism takes place when a sufficient quantity of excited hydrogen is present in the thermosphere and the planet is irradiated with intense stellar near-ultraviolet radiation. In that case, the energy absorbed in the thermosphere from the stellar Balmer irradiation exceeds the one absorbed from the stellar high-energy EUV irradiation. Thus, the thermosphere undergoes a stronger heating and expansion, leading to a higher mass-loss rate, even if the heating efficiency stays moderate. The measured mass-loss rate for WASP-33b is $\dot{\mathrm{M}}=10^{11.8_{-0.5}^{+0.6}} \mathrm{~g} \mathrm{~s}^{-1}$, while that of KELT-9b is $\dot{M}=10^{12.8 \pm 0.3} \mathrm{~g} \mathrm{~s}^{-1}$ (Wyttenbach et al. 2020). These measurements are compatible with a "Balmer-driven" evaporation, since WASP-33b orbits an A5 star, while KELT-9b orbits an A0V star, where the Balmer flux is extremely high.

\section{Conclusions}

We observed four transits of the ultra-hot Jupiter WASP-33b with the CARMENES and HARPS-N spectrographs. After the correction of the RM and CLV effects, we detected the Balmer $\mathrm{H} \alpha, \mathrm{H} \beta$, and $\mathrm{H} \gamma$ transmission spectra of the planetary atmosphere. The combined $\mathrm{H} \alpha$ transmission spectrum has a large absorption depth of $0.99 \pm 0.05 \%$, indicating that the line probes neutral hydrogen atoms in the high-altitude thermosphere. Although the detection of the Balmer lines is unambiguous, the strengths of the lines are affected by the stellar pulsation. Future modelling and correction of the spectral pulsation feature will enable a better constrain the line strength.

We fitted the observed Balmer lines using the PAWN model assuming that the atmosphere is hydrodynamic and in LTE. The model fit returns a thermospheric temperature of $T=12200_{-1000}^{+1300}$

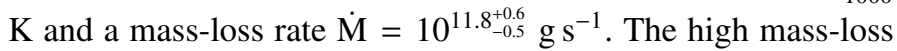
rate is consistent with theoretical predictions for UHJs orbiting early type stars (e.g., Fossati et al. 2018; García Muñoz \& Schneider 2019).

The Balmer lines have so far been detected in five ultrahot Jupiters (KELT-9b, KELT-20b/MASCARA-2b, WASP-12b, WASP-121b, and WASP-33b). Balmer absorption is probably a common spectral feature in the transmission spectra of ultrahot Jupiters because their hot atmospheres are intensively irradiated by their host stars, which could produce a large number of hydrogen atoms in the excited state. However, for some UHJs, their low atmospheric scale heights (see e.g. the case of WASP-189b, Cauley et al. 2020a) or the Rossiter-McLaughlin effect (e.g., Casasayas-Barris et al. 2020) could hamper the detection of the Balmer features. Extending the observations to a larger UHJ sample will enable a systematic study of the Balmer lines and the thermospheric conditions.

Acknowledgements. We thank the referee for the useful comments. F.Y. acknowledges the support of the DFG priority program SPP 1992 "Exploring the Diversity of Extrasolar Planets (RE 1664/16-1)". CARMENES is an instrument for the Centro Astronómico Hispano-Alemán (CAHA) at Calar Alto (Almería, Spain), operated jointly by the Junta de Andalucía and the Instituto de Astrofísica de Andalucía (CSIC). CARMENES was funded by the Max-Planck-Gesellschaft (MPG), the Consejo Superior de Investigaciones Científicas (CSIC), the Ministerio de Economía y Competitividad (MINECO) and the European Regional Development Fund (ERDF) through projects FICTS-2011-02, ICTS-2017-07CAHA-4, and CAHA16-CE-3978, and the members of the CARMENES Consortium (Max-Planck-Institut für Astronomie, Instituto de Astrofísica de Andalucía, Landessternwarte Königstuhl, Institut de Ciències de l'Espai, Institut für Astrophysik Göttingen, Universidad Complutense de Madrid, Thüringer Landessternwarte Tautenburg, Instituto de Astrofísica de Canarias, Hamburger Sternwarte, Centro de Astrobiología and Centro Astronómico Hispano-Alemán), with additional contributions by the MINECO, the Deutsche Forschungsgemeinschaft through the Major Research Instrumentation Programme and Research Unit FOR2544 "Blue Planets around Red Stars", the Klaus Tschira Stiftung, the states of Baden-Württemberg and Niedersachsen, and by the Junta de Andalucía. Based on data from the CARMENES data archive at CAB (CSICINTA). We acknowledge financial support from the Agencia Estatal de Investigación of the Ministerio de Ciencia, Innovación y Universidades and the ERDF through projects PID2019-109522GB-C51/2/3/4, PGC2018-098153-BC33, AYA2016-79425-C3-1/2/3-P, ESP2016-80435-C2-1-R and the Centre of Excellence "Severo Ochoa" and "María de Maeztu" awards to the Instituto de Astrofísica de Canarias (SEV-2015-0548), Instituto de Astrofísica de Andalucía (SEV-2017-0709), and Centro de Astrobiología (MDM-2017-0737), and the Generalitat de Catalunya/CERCA programme. A.W. acknowledges the financial support of the SNSF by grant number P400P2_186765. P.M. acknowledges support from the European Research Council under the Horizon 2020 Framework 


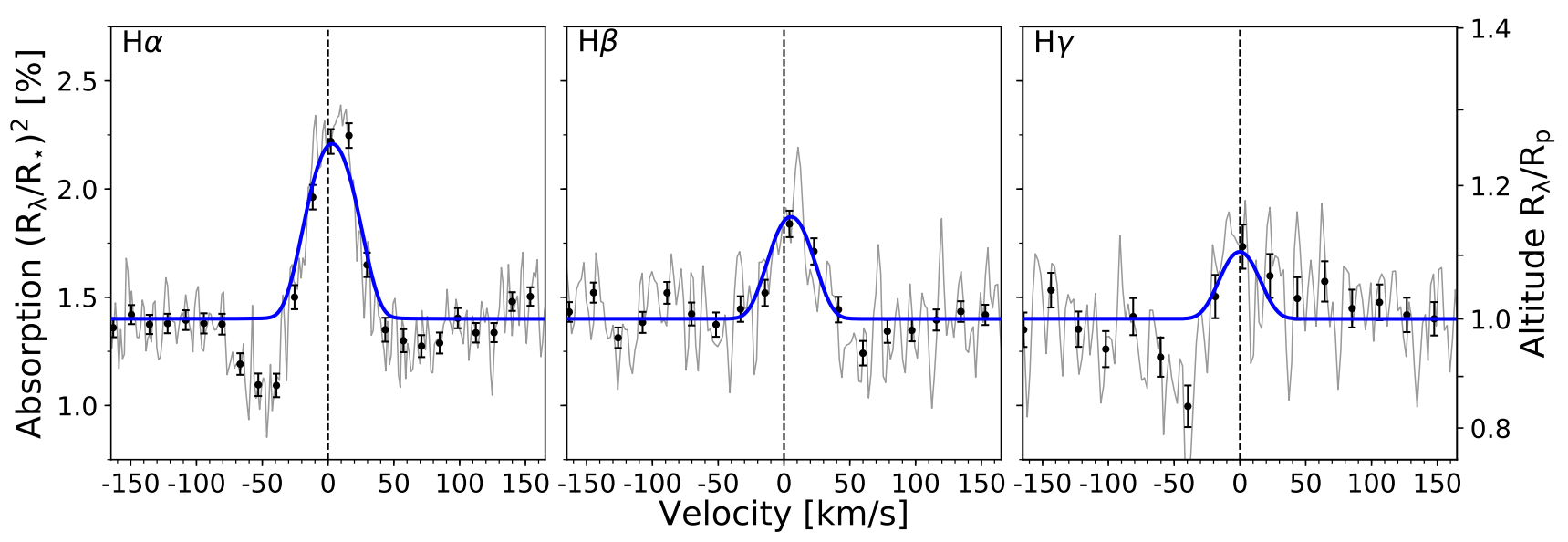

Fig. 9. Best-fit PAWN models (blue lines) and the observed transmission spectra (grey lines and black points) in the planetary rest frame. The PAWN models are for the case of a hydrodynamically expanding atmosphere in LTE.

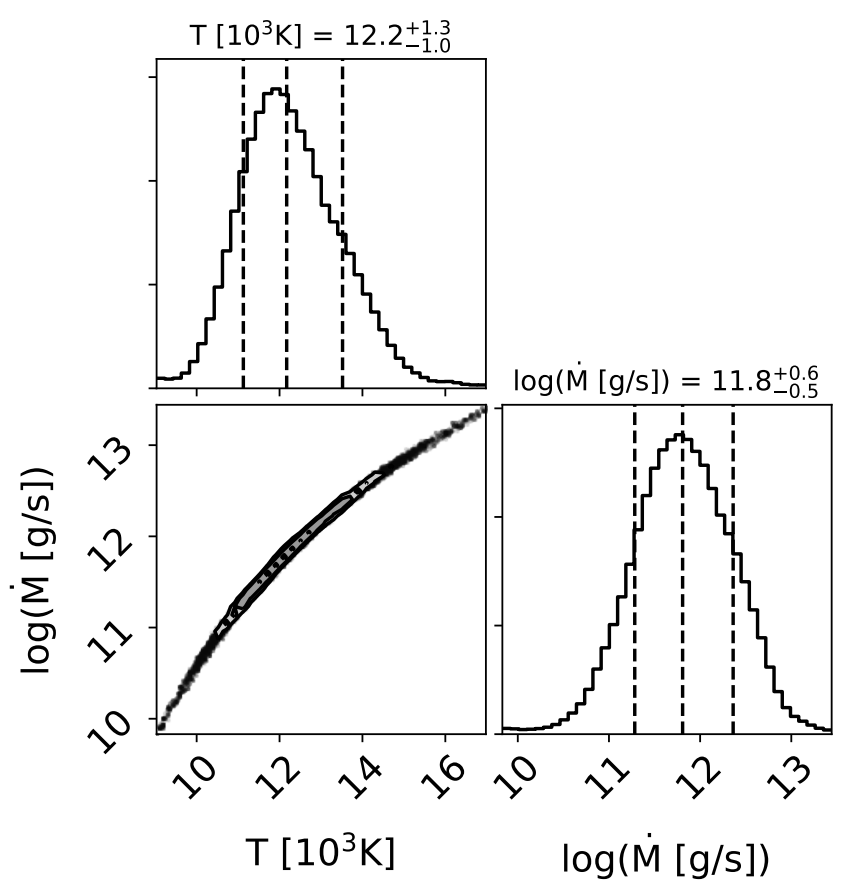

Fig. 10. Correlation diagram of the MCMC posterior distributions in the case of a hydrodynamic atmosphere in LTE. The result is for the combined fit of all Balmer lines from HARPS-N and CARMENES. The retrieved parameters are the thermospheric temperature $\left(T=12200_{-1000}^{+1300}\right.$ $\mathrm{K})$ and the atmospheric mass-loss rate $\left(\dot{\mathrm{M}}=10^{11.8_{-0.5}^{+0.6}} \mathrm{~g} \mathrm{~s}^{-1}\right)$.

Program via ERC grant 832428. I.S. acknowledges funding from the European Research Council (ERC) under the European Union's Horizon 2020 research and innovation program under grant agreement No 694513. M.L. achkowledges the funding from the project ESP2017-87143-R. This work is based on observations made with the Italian Telescopio Nazionale Galileo (TNG) operated on the island of La Palma by the Fundación Galileo Galilei of the INAF (Istituto Nazionale di Astrofisica) at the Spanish Observatorio del Roque de los Muchachos of the Instituto de Astrofisica de Canarias.

\section{References}

Allart, R., Bourrier, V., Lovis, C., et al. 2018, Science, 362, 1384 Arcangeli, J., Désert, J.-M., Line, M. R., et al. 2018, ApJ, 855, L30

Barnes, J. R., Haswell, C. A., Staab, D., \& Anglada-Escudé, G. 2016, MNRAS, 462,1012
Bell, T. J. \& Cowan, N. B. 2018, ApJ, 857, L20

Ben-Yami, M., Madhusudhan, N., Cabot, S. H. C., et al. 2020, ApJ, 897, L5

Bourrier, V., Ehrenreich, D., Lendl, M., et al. 2020a, A\&A, 635, A205

Bourrier, V., Kitzmann, D., Kuntzer, T., et al. 2020b, A\&A, 637, A36

Brogi, M., de Kok, R. J., Albrecht, S., et al. 2016, ApJ, 817, 106

Cabot, S. H. C., Madhusudhan, N., Welbanks, L., Piette, A., \& Gandhi, S. 2020 , MNRAS, 494, 363

Casasayas-Barris, N., Pallé, E., Yan, F., et al. 2018, A\&A, 616, A151

Casasayas-Barris, N., Pallé, E., Yan, F., et al. 2019, A\&A, 628, A9

Casasayas-Barris, N., Pallé, E., Yan, F., et al. 2020, A\&A, 635, A206

Cauley, P. W., Redfield, S., \& Jensen, A. G. 2017a, AJ, 153, 217

Cauley, P. W., Redfield, S., \& Jensen, A. G. 2017b, AJ, 153, 185

Cauley, P. W., Redfield, S., Jensen, A. G., \& Barman, T. 2016, AJ, 152, 20

Cauley, P. W., Shkolnik, E. L., Ilyin, I., et al. 2019, AJ, 157, 69

Cauley, P. W., Shkolnik, E. L., Ilyin, I., et al. 2020a, Research Notes of the American Astronomical Society, 4, 53

Cauley, P. W., Wang, J., Shkolnik, E. L., et al. 2020b, arXiv e-prints, arXiv:2010.02118

Chen, G., Casasayas-Barris, N., Pallé, E., et al. 2020, A\&A, 635, A171

Christie, D., Arras, P., \& Li, Z.-Y. 2013, ApJ, 772, 144

Collier Cameron, A., Guenther, E., Smalley, B., et al. 2010, MNRAS, 407, 507

Cubillos, P. E., Fossati, L., Koskinen, T., et al. 2020, AJ, 159, 111

Czesla, S., Klocová, T., Khalafinejad, S., Wolter, U., \& Schmitt, J. H. M. M. 2015, A\&A, 582, A51

Daylan, T., Günther, M. N., Mikal-Evans, T., et al. 2019, arXiv e-prints, arXiv: 1909.03000

Ehrenreich, D., Bourrier, V., Wheatley, P. J., et al. 2015, Nature, 522, 459

Ehrenreich, D., Lovis, C., Allart, R., et al. 2020, Nature

Foreman-Mackey, D., Hogg, D. W., Lang, D., \& Goodman, J. 2013, PASP, 125, 306

Fossati, L., Haswell, C. A., Froning, C. S., et al. 2010, ApJ, 714, L222

Fossati, L., Koskinen, T., Lothringer, J. D., et al. 2018, ApJ, 868, L30

García Muñoz, A. \& Schneider, P. C. 2019, ApJ, 884, L43

Gibson, N. P., Merritt, S., Nugroho, S. K., et al. 2020, MNRAS, 220

Haynes, K., Mandell, A. M., Madhusudhan, N., Deming, D., \& Knutson, H. 2015, ApJ, 806, 146

Helling, C., Gourbin, P., Woitke, P., \& Parmentier, V. 2019, A\&A, 626, A133

Hoeijmakers, H. J., Cabot, S. H. C., Zhao, L., et al. 2020, A\&A, 641, A120

Hoeijmakers, H. J., Ehrenreich, D., Heng, K., et al. 2018, Nature, 560, 453

Hoeijmakers, H. J., Ehrenreich, D., Kitzmann, D., et al. 2019, A\&A, 627, A165

Huang, C., Arras, P., Christie, D., \& Li, Z.-Y. 2017, ApJ, 851, 150

Iorio, L. 2016, MNRAS, 455, 207

Jensen, A. G., Cauley, P. W., Redfield, S., Cochran, W. D., \& Endl, M. 2018, AJ, 156,154

Jensen, A. G., Redfield, S., Endl, M., et al. 2012, ApJ, 751, 86

Johnson, M. C., Cochran, W. D., Collier Cameron, A., \& Bayliss, D. 2015, ApJ, 810, L23

Kesseli, A. Y., Snellen, I. A. G., Alonso-Floriano, F. J., Mollière, P., \& Serindag, D. B. 2020, AJ, 160, 228

Kitzmann, D., Heng, K., Rimmer, P. B., et al. 2018, ApJ, 863, 183

Komacek, T. D. \& Tan, X. 2018, Research Notes of the American Astronomical Society, 2, 36

Kovács, G., Kovács, T., Hartman, J. D., et al. 2013, A\&A, 553, A44

Kreidberg, L., Line, M. R., Parmentier, V., et al. 2018, AJ, 156, 17

Kurucz, R. L. 1979, ApJS, 40, 1 
Kurucz, R. L. 1992, in IAU Symposium, Vol. 149, The Stellar Populations of Galaxies, ed. B. Barbuy \& A. Renzini, 225

Lampón, M., López-Puertas, M., Lara, L. M., et al. 2020, A\&A, 636, A13

Lecavelier des Etangs, A., Bourrier, V., Wheatley, P. J., et al. 2012, A\&A, 543, L4

Lecavelier Des Etangs, A., Pont, F., Vidal-Madjar, A., \& Sing, D. 2008, A\&A, 481, L83

Lehmann, H., Guenther, E., Sebastian, D., et al. 2015, A\&A, 578, L4

Lothringer, J. D., Barman, T., \& Koskinen, T. 2018, ApJ, 866, 27

Louden, T. \& Wheatley, P. J. 2015, ApJ, 814, L24

Mansfield, M., Bean, J. L., Line, M. R., et al. 2018, AJ, 156, 10

Mansfield, M., Bean, J. L., Stevenson, K. B., et al. 2020, ApJ, 888, L15

Mollière, P., van Boekel, R., Bouwman, J., et al. 2017, A\&A, 600, A10

Mollière, P., Wardenier, J. P., van Boekel, R., et al. 2019, A\&A, 627, A67

Nortmann, L., Pallé, E., Salz, M., et al. 2018, Science, 362, 1388

Nugroho, S. K., Gibson, N. P., de Mooij, E. J. W., et al. 2020a, ApJ, 898, L31

Nugroho, S. K., Gibson, N. P., de Mooij, E. J. W., et al. 2020b, MNRAS, 496, 504

Nugroho, S. K., Kawahara, H., Masuda, K., et al. 2017, AJ, 154, 221

Owen, J. E. 2019, Annual Review of Earth and Planetary Sciences, 47, 67

Palle, E., Nortmann, L., Casasayas-Barris, N., et al. 2020, A\&A, 638, A61

Parmentier, V., Line, M. R., Bean, J. L., et al. 2018, A\&A, 617, A110

Pino, L., Désert, J.-M., Brogi, M., et al. 2020, ApJ, 894, L27

Queloz, D., Eggenberger, A., Mayor, M., et al. 2000, A\&A, 359, L13

Quirrenbach, A., Amado, P. J., Ribas, I., et al. 2018, in Society of Photo-Optical Instrumentation Engineers (SPIE) Conference Series, Vol. 10702, Proc. SPIE, $107020 \mathrm{~W}$

Salz, M., Czesla, S., Schneider, P. C., et al. 2018, A\&A, 620, A97

Salz, M., Czesla, S., Schneider, P. C., \& Schmitt, J. H. M. M. 2016, A\&A, 586, A75

Sharp, C. M. \& Burrows, A. 2007, ApJS, 168, 140

Sing, D. K., Lavvas, P., Ballester, G. E., et al. 2019, AJ, 158, 91

Snellen, I. A. G., de Kok, R. J., de Mooij, E. J. W., \& Albrecht, S. 2010, Nature, 465,1049

Spake, J. J., Sing, D. K., Evans, T. M., et al. 2018, Nature, 557, 68

Stangret, M., Casasayas-Barris, N., Pallé, E., et al. 2020, A\&A, 638, A26

Stevenson, K. B., Bean, J. L., Madhusudhan, N., \& Harrington, J. 2014, ApJ, 791,36

Tian, F., Toon, O. B., Pavlov, A. A., \& De Sterck, H. 2005, ApJ, 621, 1049

Turner, J. D., de Mooij, E. J. W., Jayawardhana, R., et al. 2020, ApJ, 888, L13

Valyavin, G. G., Gadelshin, D. R., Valeev, A. F., et al. 2018, Astrophysical Bulletin, 73,225

Vidal-Madjar, A., Lecavelier des Etangs, A., Désert, J.-M., et al. 2003, Nature, 422,143

von Essen, C., Czesla, S., Wolter, U., et al. 2014, A\&A, 561, A48

von Essen, C., Mallonn, M., Borre, C. C., et al. 2020, A\&A, 639, A34

von Essen, C., Mallonn, M., Welbanks, L., et al. 2019, A\&A, 622, A71

Watanabe, N., Narita, N. \& Johnson, M. C. 2020, PASJ

Wong, I., Shporer, A., Kitzmann, D., et al. 2020, AJ, 160, 88

Wyttenbach, A., Ehrenreich, D., Lovis, C., Udry, S., \& Pepe, F. 2015, A\&A, 577, A62

Wyttenbach, A., Mollière, P., Ehrenreich, D., et al. 2020, A\&A, 638, A87

Yan, F., Casasayas-Barris, N., Molaverdikhani, K., et al. 2019, A\&A, 632, A69

Yan, F., Fosbury, R. A. E., Petr-Gotzens, M. G., Zhao, G., \& Pallé, E. 2015a, A\&A, 574, A94

Yan, F., Fosbury, R. A. E., Petr-Gotzens, M. G., et al. 2015b, International Journal of Astrobiology, 14, 255

Yan, F. \& Henning, T. 2018, Nature Astronomy, 2, 714

Yan, F., Pallé, E., Fosbury, R. A. E., Petr-Gotzens, M. G., \& Henning, T. 2017, A\&A, 603, A73

Yan, F., Pallé, E., Reiners, A., et al. 2020, A\&A, 640, L5

Yelle, R. V. 2004, Icarus, 170, 167

Zhang, M., Knutson, H. A., Kataria, T., et al. 2018, AJ, 155, 83 
F. Yan et al.: Detection of the hydrogen Balmer lines in the ultra-hot Jupiter WASP-33b

\section{Appendix A: Additional figures}



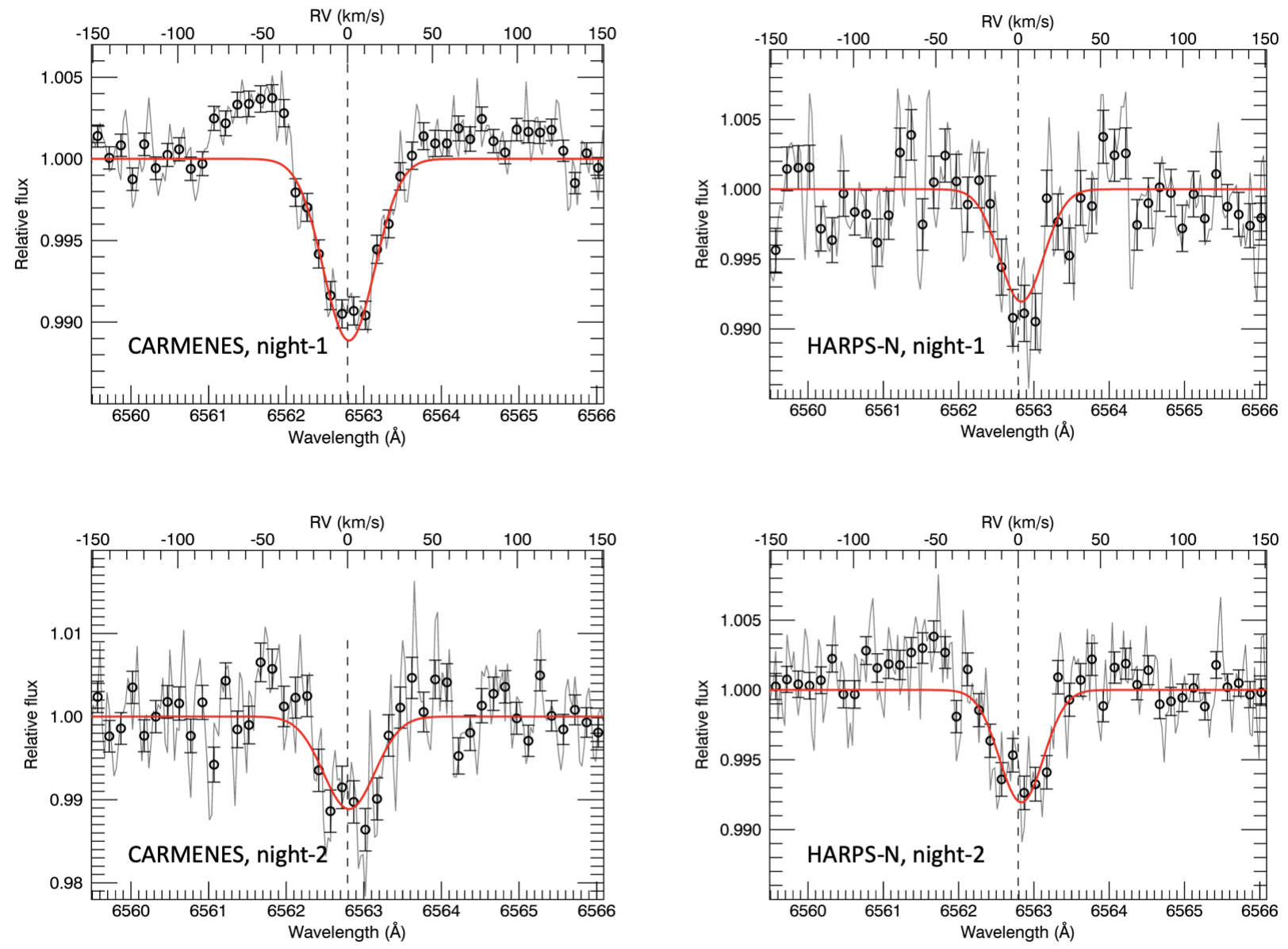

Fig. A.1. $\mathrm{H} \alpha$ transmission spectra of the four nights. The grey lines are the original spectra and the black points are spectra binned every five points. The red lines are the same best-fit Gaussian functions as in Fig. 4. 